\title{
La universidad argentina resiliente, ¿nuevos escenarios en el horizonte?
}

\author{
MARTín UnZUÉ
}

Doctor en Ciencias Sociales. Profesor investigador de la Universidad de Buenos Aires y de la Universidad Nacional de La Plata. Actualmente es director del Instituto de Investigaciones Gino Germani de la Facultad de Ciencias Sociales de la UBA.

\begin{abstract}
Resumen
El artículo analiza los escenarios que se abren para la universidad argentina con el cambio de gobierno sucedido a fines del año 2019. Luego de repasar las principales medidas tomadas por el anterior gobierno, tanto en políticas de educación superior como de ciencia y tecnología, se hace un análisis de lo que se insinúa como las nuevas líneas de acción para el sector. Se plantea que en el actual contexto de incertidumbre esa nueva agenda aún no está plenamente definida ni abordada y que limitaciones de diversa índole, en especial las presupuestarias, operarán en el mediano plazo, amenazando fuertemente la posibilidad de instrumentar políticas que salden algunas de las grandes deudas vigentes con las universidades argentinas.
\end{abstract}

Palabras clave: Universidad; políticas públicas; Argentina; gobierno.

\section{A universidade argentina resiliente, novos cenários no horizonte?}

Resumo

O artigo analisa os cenários que se abrem para a universidade argentina com a mudança de governo ocorrida no final de 2019. Após analisar as principais medidas adotadas pelo governo anterior, tanto nas políticas de ensino superior quanto nas de ciência e tecnologia, é feito. uma análise do que está sendo sugerido como novas linhas de ação para o setor. Argumenta-se que, no atual contexto de incerteza, essa nova agenda ainda não foi totalmente definida ou abordada e que limitações de vários tipos, especialmente as orçamentárias, funcionarão plenamente no médio prazo, ameaçando fortemente a possibilidade de fazer políticas que resolvam algumas das grandes dívidas pendentes com as universidades argentinas.

Palavras chave: Universidade; políticas públicas; Argentina; governo.

DOI: https://doi.org/10.36888/udual.universidades.2020.85.277 


\title{
The Resilient Argentinian University, New Scenarios on The Horizon?
}

\begin{abstract}
This research analyzes the scenarios in the Argentina university field that came with the change of government at the end of 2019. After reviewing the main measures taken by the previous government, in higher education and in science and technology policies, we did an examination of what new lines of action were hints in this sector. It is argued that in the current context of uncertainty, the new agenda has not yet been fully defined or addressed, and that limitations of different nature, especially the budgetary ones, will fully operate in medium term, which strongly threatens the possibility of making policies that find the solution to some of the large outstanding debts with Argentinian universities.
\end{abstract}

Keywords: University; public policies; Argentina; government.

\section{Introducción}

El sistema universitario argentino ha reunido una serie de características a lo largo de su historia del último siglo. Allí, ciertos hitos como la Reforma Universitaria de 1918 o las intervenciones militares que se fueron reiterando con grados de violencia significativos, particularmente luego de los golpes de estado de 1966 y 1976, le dieron una identidad que comenzó a tomar su actual forma desde fines de 1983, a partir del retorno a la democracia.

Esa identidad no significa que se trate de un sistema homogéneo, ni siquiera dentro del subsector más relevante, formado actualmente por las 57 universidades nacionales. Pero sí que hay algunas características, como la gratuidad de los estudios de grado, el cogobierno y la autonomía, que se han ido consolidando en esas instituciones. La autonomía resulta de singular relevancia pues las universidades públicas argentinas parecen desplegar un sentido fuerte de este término, aunque no todas las instituciones tienen las mismas capacidades para su ejercicio.

La incorporación de la autonomía universitaria al texto constitucional con la reforma de 1994, si bien no es original, le otorga una singular relevancia al tema al ponerlo en la cúspide del sistema normativo ${ }^{1}$.

Pero ello no significa que las universidades escapen al contexto sociopolítico nacional o a las condiciones macroeconómicas, en especial cuando algunas de las severas crisis que han azotado al país en estos tiempos, como la hiperinflacionaria de 1989-90 o la crisis sistémica de fines de 2001, y seguramente podremos poner en la lista a la actual de $2020^{2}$, han conmovido 
profundamente el funcionamiento del sistema universitario de modos muy diversos.

Por ello las universidades argentinas ya han mostrado una gran resiliencia en su historia más reciente.

\section{Algunas características del sistema}

El sistema universitario argentino es un sistema masivo que se caracteriza por su elevado nivel de cobertura tanto en términos de población como de geografía ${ }^{3}$, y una creciente complejidad y diversidad de sus instituciones integrantes.

Destaca, en el contexto regional, el predominio de su subsistema público, ampliamente mayoritario más que en número de establecimientos, en cantidad de estudiantes (78\% de los estudiantes de grado pertenecen a instituciones estatales).

La gratuidad de los estudios de grado ${ }^{4}$, sumado a la ausencia de limitaciones importantes para el ingreso a las universidades nacionales ${ }^{5}$, se ha presentado como límite para el desarrollo de un sector privado de educación superior.

La máxima que "nadie pagaría por lo que puede tener gratuitamente" ha funcionado con relativo éxito por algunas décadas, al menos desde 1958 cuando las universidades privadas fueron autorizadas a emitir títulos habilitantes.

El proceso de neoliberalización de alcance mundial, que se ha ido consolidando localmente, comenzó a erosionar esa idea. El avance de la privatización en los niveles educativos previos (primario y secundario) resultó un antecedente importante para permitir que el sector universitario privado comience a crecer en su participación, al menos en las últimas dos décadas ${ }^{6}$.

Para ciertos sectores sociales pagar por la educación se ha vuelto algo normal, como consecuencia de la degradación de algunos servicios públicos que produce la prolongada crisis fiscal. Esto terminó generando una valoración de lo privado que no conoce en el sistema universitario la misma fortaleza que en otros sectores, pero que opera en modos tangenciales.

Dicho esto, ni el subsistema público o el privado son homogéneos en su interior, existiendo en ambos condiciones de funcionamiento y calidades académicas diversas.

Podemos decir, en términos generales, que el sistema universitario argentino es predominantemente profesionalista. Las carreras llamadas "tradicionales"7, 


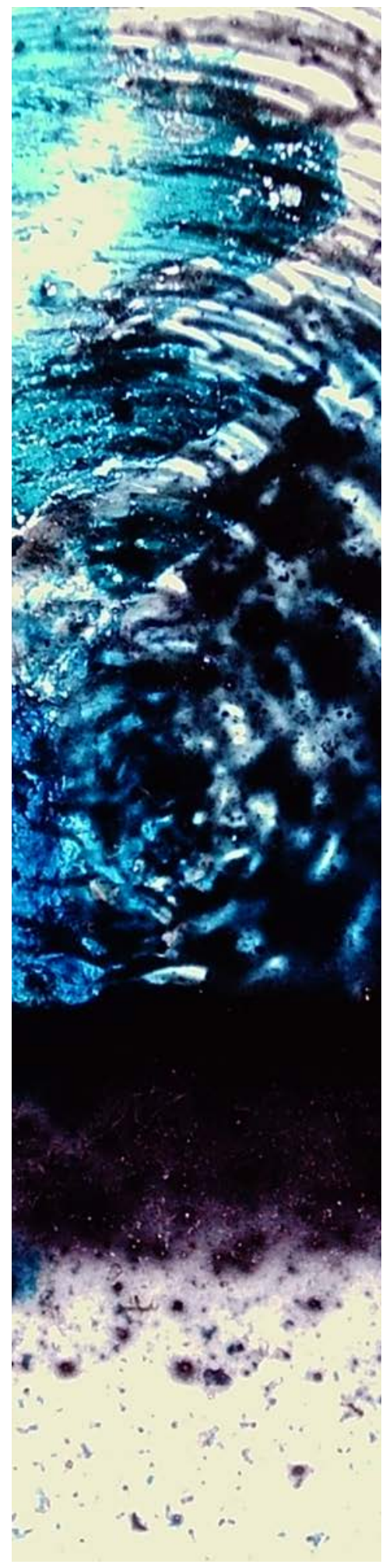

siguen siendo las más elegidas por los ingresantes, en parte como resultado de una impronta liberal que ha dificultado los intentos de orientación de la matrícula con base en la definición de áreas de vacancia o de desarrollo estratégico ${ }^{8}$.

Este sistema entonces, y en especial el público, inscribe a los estudiantes con requisitos mínimos (haber terminado los estudios medios), lo que se traduce en un elevado nivel de acceso a la educación universitaria en comparación con otros países de desarrollo similar.

Algo distinto es lo que vemos con la graduación, en donde los niveles de deserción concentrados en muchos casos en el primer año producen tasas de titulación bajas. Hay mucho estudiado sobre este fenómeno? ${ }^{9}$.

En relación con los posgrados, los mismos han crecido fuertemente desde finales de los 90 , concentrados en especializaciones y maestrías de carácter profesionalizante. En los últimos quince años ese proceso también se ha verificado al nivel de los doctorados, al menos en algunas disciplinas en las que no había una fuerte tradición de estudios doctorales y posdoctorales, y que se han ido consolidando de forma veloz.

Esto va de la mano con los procesos de reorganización de la llamada "carrera académica" que se van produciendo desde los 90, y que conocen una nueva etapa a partir de mediados de la década pasada ${ }^{10}$.

Buena parte de esa carrera académica ha estado orientada a desarrollar tareas de investigación científica por parte de los profesores universitarios.

Las universidades, y en especial las públicas, han sido y siguen siendo esenciales en el sistema científico argentino, tanto como lugar de trabajo de la gran mayoría de sus investigadores, como en su rol de formadoras de nuevos científicos e incluso, aunque con limitaciones, en el financiamiento de esos trabajos. No obstante, la distribución de la investigación en el sistema universitario argentino no es muy homogénea, distinguiéndose ciertas concentraciones por tipo de universidad y por disciplina.

Finalmente podemos decir que el sistema también se caracteriza por el predominio de docentes que no se dedican en forma exclusiva a sus tareas universitarias o que no lo hacen en una sola casa de estudios $^{11}$.

Esto, si varía fuertemente por tipo de universidad y disciplina, también se presenta como la constante 
en la gran mayoría de las instituciones públicas y privadas y resulta un importante límite.

\section{Las universidades argentinas durante el anterior ciclo presidencial}

El gobierno que encabezó Mauricio Macri asume la presidencia de Argentina en diciembre de $2015^{12}$.

Se trata de un autoreferido "cambio de rumbo". El triunfo opositor, a través de una coalición de partidos bajo el nombre de Cambiemos ${ }^{13}$, viene a replantear varias de las principales políticas que se habían desplegado en los años previos, lo que incluye las relativas a la educación superior.

A raíz de algunas de las medidas económicas tomadas inicialmente ${ }^{14}$ se produjo una aceleración de la inflación ya existente. Esto se tradujo en una desactualización o caída en términos reales de presupuestos y salarios del sector universitario ${ }^{15}$.

El resultado de lo segundo fue un creciente número de huelgas a partir de 2016, iniciando un ciclo de fuerte conflictividad laboral a lo largo de todo el periodo ${ }^{16}$.

A ello se le fueron agregando diversas subejercicios de partidas presupuestarias ya aprobadas, en general vinculadas a diversos programas específicos que se interrumpieron, dejando obras de infraestructura demoradas, inconclusas o canceladas.

A tan solo dos meses de su asunción, el presidente dicta el decreto núm. 336/2016 que suspende la mayor parte de los contratos entre el Estado Nacional y las universidades públicas. Se trata de la reversión parcial de una norma dictada en el $2001^{17}$, que permitía al Estado Nacional contratar en forma directa con las universidades nacionales, y que había, por un lado, facilitado los procesos de ejecución de asistencia técnica pero que además, se traducía en una ventaja de esas universidades para captar recursos surgidos de tareas de asesoramiento que el Estado contrata de modo tercerizado en forma habitual.

La lectura de los considerandos del mencionado decreto deja en claro su espíritu. Allí se sostiene que se debe modificar la política de contratación de personal por parte del Estado, apelando a criterios de racionalidad y eficiencia, pero también hace referencia a la transparencia de esas contrataciones, sembrando una sombra de duda sobre los mecanismos de control y auditoría operantes en estos casos.

Como síntesis, el gobierno suspende la absoluta mayoría de los contratos vigentes con las universidades públicas ${ }^{18}$, crea un registro para los que permanezcan vigentes, y pone en manos de la Sindicatura General de la Nación (SIGEN) la realización de una amplia auditoría sobre las contrataciones realizadas en el último cuatrimestre de 2015 .

Pocos meses después, un fiscal federal presenta una masiva denuncia penal contra 52 universidades nacionales, es decir, casi la totalidad de las universidades en funcionamiento ${ }^{19}$, para que se investigue una supuesta malversación de caudales públicos derivada de esos convenios. 


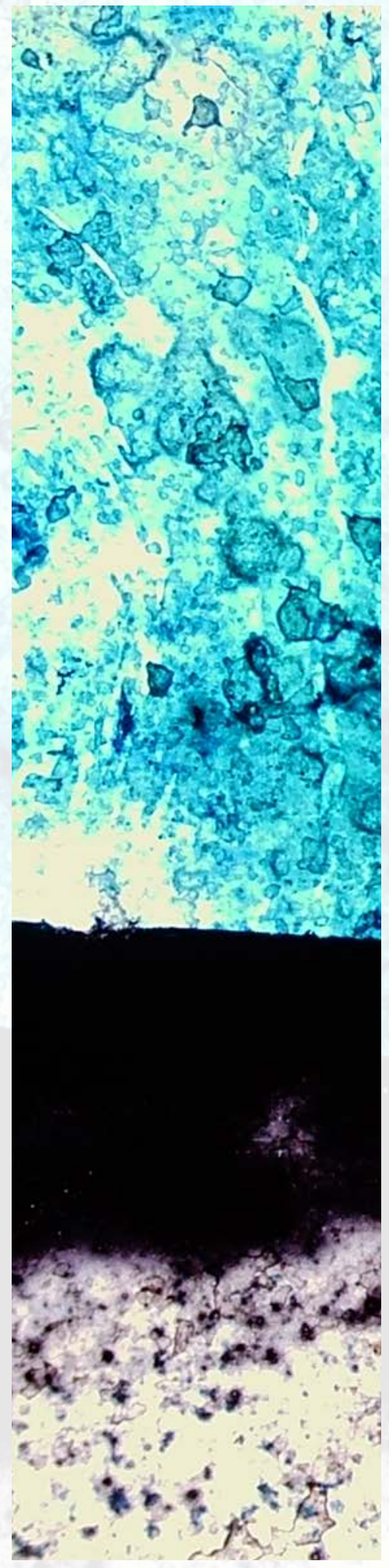

Esta secuencia comienza a marcar la relación del sistema universitario con el gobierno asumido en diciembre de 2015: a los reclamos presupuestarios, le siguen los reclamos políticos por las suspensiones de convenios y subejecuciones presupuestarias, y luego por las denuncias en sede judicial. En paralelo los gremios docentes y de personal administrativo llevan adelante permanentes reclamos por la desvalorización de los salarios.

Esto se extiende al resto del sistema científico-tecnológico, aunque toma particular visibilidad en el caso del Consejo Nacional de Investigaciones Científicas y Técnicas (CONICET) que funciona muy articulado con el sistema universitario, en especial, por compartir frecuentemente personal, sedes e infraestructura fundamental.

Luego de un periodo de fuerte crecimiento del organismo, que se expresó en mayor presupuesto, y un inédito incremento de los fondos para becas doctorales y postdoctorales y para contratación de investigadores que comenzaron a ingresar como personal de planta al organismo ${ }^{20}$, eso se interrumpe con la nueva gestión gubernamental, llevando a un severo y prolongado conflicto ya desde finales de 2016.

La reducción de plazas para postulantes, que habían sido recomendados por las comisiones evaluadoras, dejó un estimado de 500 científicos sin ingreso al organismo, lo que derivó en una fuerte movilización de la comunidad científica y universitaria, que logró una importante visibilidad pública.

Recordemos que el tema de la política para el sector de la ciencia tuvo la particularidad de ser el único en el que la gestión del nuevo gobierno pretendió sostener una continuidad con su antecesor, expresada tanto en la campaña electoral en la que el candidato Macri manifestó en reiteradas oportunidades su voluntad de continuar con el apoyo al crecimiento dentro del sector científico, como en la inédita continuidad del mismo ministro del área ante el cambio de gobierno ${ }^{21}$.

Sin embargo, a poco de iniciarse el nuevo gobierno, esas promesas se volvieron incumplidas. Tanto a fines de 2016 como durante 2017 y comienzos del 2018, los sucesivos conflictos presupuestarios dieron lugar a huelgas en el sector científico e incluso a la reiterada toma pacífica de la sede central del Ministerio de Ciencia y Tecnología en reclamo por el mantenimiento de las cantidades de ingresos al CONICET, previstas en los planes nacionales de ciencia,

70 Universidades $\mid$ núm. 85, julio-septiembre 2020 | UDUAL | DOI:https://doi.org/10.36888/udual.universidades.2020.85.277 Martín Unzué | La universidad argentina resiliente, ¿̇nuevos escenarios en el horizonte? 
así como de las becas doctorales y posdoctorales y por la actualización de los salarios fuertemente devaluados.

La respuesta del gobierno ante el conflicto será mantener su posición de no incorporar a esos científicos excluidos del ingreso a fines de 2016, aunque sí habilitar un mecanismo ad hoc de otorgamiento de cargos docentes con dedicaciones exclusivas en universidades nacionales.

El lanzamiento del PRIDIUN, Programa de Incorporación de Docentes Investigadores a las Universidades Nacionales, como modo de desarticular la crisis, contempla la casi totalidad de los postulantes relegados (480 altas), estableciendo un mecanismo extraordinario que no se repetiría en los años posteriores.

La solución dada a la crisis de 2016 no resultó óptima desde el punto de vista de los investigadores afectados, que se vieron forzados a cambiar una carrera científica con claros procesos de promoción, por un cargo docente sin esa perspectiva, y tampoco para la ciencia en general al ser un reemplazo de investigadores de tiempo completo, por docentes universitarios investigadores, con obligaciones en las dos funciones.

Pero para las universidades nacionales que acogieron a esos investigadores desairados significó la poco frecuente posibilidad de nombrar docentes con cargos exclusivos, recibiendo los recursos para ello, aunque sin la potestad de seleccionarlos.

A la ya referida falta de dedicaciones exclusivas en el sistema universitario argentino se le dio una respuesta indirecta, que aunque beneficiosa para el conjunto de las instituciones públicas, fue poco significativa en número ${ }^{22}$, no tuvo continuidad en otros años y no resultó de una verdadera voluntad de fortalecimiento de las capacidades de investigación de las universidades, sino de la urgencia de hacer una concesión puntual para acallar un problema que estaba teniendo altos costos en términos de imagen del gobierno.

Vale notar que estas políticas de ajuste fiscal que estamos presentando no tuvieron impactos homogéneos a lo largo de todo el sistema universitario público. Las vinculaciones políticas entre gestiones universitarias y ministeriales, o la visibilidad de algunas casas de estudios, lograron ponerlas relativamente al margen de ciertas restricciones, mientras a otras, en particular las universidades más recientes en proceso de consolidación, con la consecuente expansión

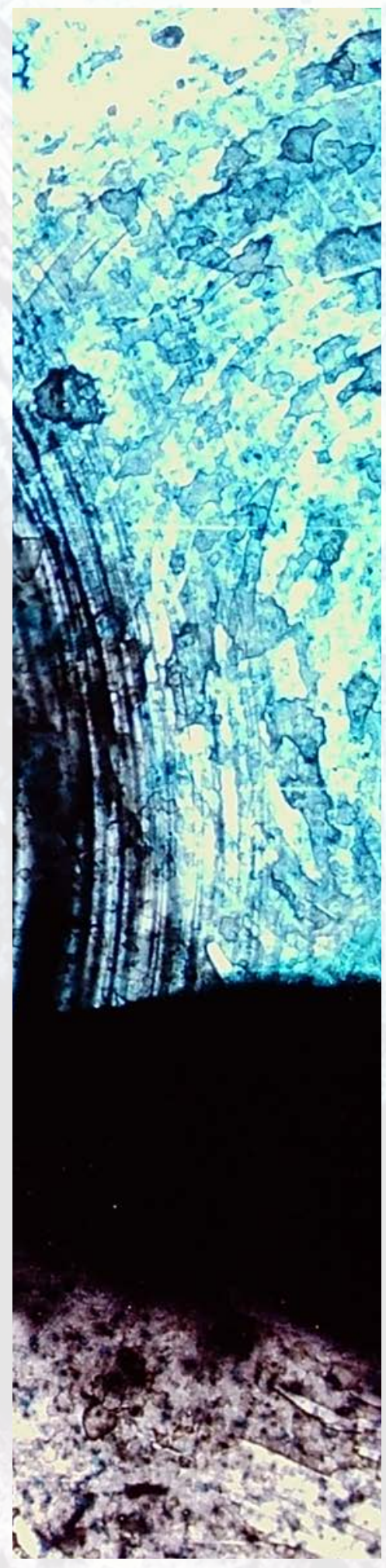


que implica tener muchas carreras nuevas abriendo cursos cada año para su primera promoción, el peso de los ajustes les resultó mucho más significativo.

Otro de los ejes de las políticas implementadas hacia el sistema universitario fue el lanzamiento, a fines de $2016^{23}$, del llamado Sistema Nacional de Reconocimiento Académico de Educación Superior.

Se trató de un esfuerzo por generar mecanismos de integración y articulación entre diversas instituciones del sistema, tanto públicas como privadas, con el fin de avanzar hacia futuros acuerdos de reconocimientos mutuos de estudios.

El sistema se presentó como de adhesión voluntaria para las instituciones en nombre de la autonomía (algunas quedaron al margen), y fue concebido como un proceso a desarrollarse en un plazo de dos años, en el que se iba a trabajar con los buenos oficios de la Secretaría de Políticas Universitarias (SPU) para facilitar esos acuerdos interinstitucionales, así como para elaborar un registro centralizado de los mismos.

El esquema prometía, una vez consolidado, permitir una mayor circulación de estudiantes entre instituciones (universidades e institutos universitarios) con base en el reconocimiento de lo que se denominó Reconocimiento de trayecto formativo y que era una suerte de sistema de créditos para que los eventuales cambios de instituciones no solo se simplifiquen en sus aspectos administrativos, sino también en los costos por no reconocimiento de estudios hechos.

El sistema preveía el cambio de institución continuando la misma carrera, pero también cambios de carreras.

Si bien la adhesión formal al mismo resultó importante, con unas 75 universidades públicas y privadas, el avance en la letra chica para la implementación de esos reconocimientos fue mucho menos prometedor, despertando diversos tipos de preguntas que no quedaban claras de la propuesta. A casi tres años de su lanzamiento se habían firmado 10 acuerdos y la SPU estimaba que había tan solo 200 estudiantes usando el programa ${ }^{24}$.

A fin de cuentas y como ya hemos planteado, el sistema universitario argentino concentra la mayor parte de sus estudiantes en instituciones públicas gratuitas, por lo que algunas lecturas del Sistema vieron que podría ser una puerta para la transferencia de estudiantes de instituciones públicas a privadas, con base en la mayor agilidad de estas últimas para crear nuevas titulaciones que podrían volverlas atractivas.

En cuanto a otras disposiciones que tendieron a incidir directamente en el funcionamiento del sistema, podemos mencionar los tardíos intentos por modificar el Programa de Incentivos a Docentes Investigadores, en una apuesta por revitalizar una herramienta que se había generado en el ciclo de los años 90, para reconfigurar los esquemas de incentivos a la carrera académica de los docentes investigadores universitarios.

En este punto ya en abril de 2018 por resolución del Ministerio de Educación ${ }^{25}$ se decide abrir el procedimiento de categorización a los docentes de universidades privadas. 
Un año después, en abril de 2019, a pocos meses del fin del mandato presidencial, se publica en el Boletín Oficial la normativa referida a la creación de un Sistema Nacional de Docentes Investigadores Universitarios (SIDIUN) que viene en reemplazo del Programa de Incentivos con sus modificatorias.

El nuevo SIDIUN presenta una gran cantidad de interrogantes, en especial por el proceso cerrado y con poca comunicación con la comunidad universitaria que lo precede. Lo que sí se puede ver del análisis de la norma es que borra en gran parte las distinciones entre instituciones públicas y privadas como ya se había hecho con la resolución de 2018, que crea un consejo asesor con una fuerte representación del CRUP (Consejo de Rectores de Universidades Privadas $)^{26}$, lo que hacía prever que en la futura definición de las formas de implementación de la norma se reproduciría la tendencia general a apoyar a estas instituciones de gestión privada.

Sin embargo, y al igual que en el Sistema Nacional de Reconocimiento Académico, los aspectos necesarios para su implementación, pero que no se aclaraban en la norma, eran muy numerosos.

Mientras se resolvía en parte el problema de la imprevisible fecha de apertura de las categorizaciones para los docentes, estableciendo una ventanilla permanente, no se decía nada ni sobre los recursos para llevar adelante ese proceso, y tampoco sobre el presupuesto del programa, ya que su finalidad original no era la mera categorización, sino el establecimiento de un adicional salarial para incentivar las tareas de investigación de los docentes universitarios.

Con el fin del mandato presidencial este tema queda, como los anteriormente referidos, sin una clara definición.

Es relevante agregar que la producción de datos estadísticos, necesarios para el estudio y la evaluación del funcionamiento del sistema, también sufre alteraciones e interrupciones en el periodo. La publicación de las estadísticas universitarias bajo la forma de los anuarios deja de producirse con regularidad y en los últimos dos años se interrumpe la producción o difusión de algunos de los principales indicadores del sector.

Finalmente, la reducción de la oferta de becas de posgrado en diversas agencias sumado al abatimiento por el cierre parcial de la vía de ingreso al CONI-

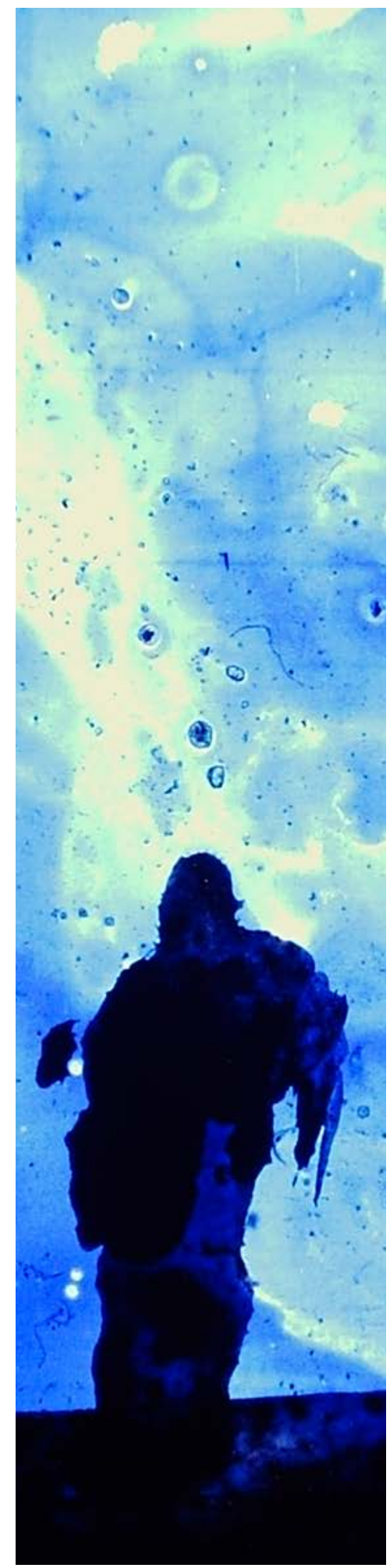


CET, que se hace evidente luego del prolongado conflicto citado, parece producir un proceso de desaliento, que impacta en los diversos doctorados que estaban en proceso de crecimiento y consolidación y que ingresan en una nueva etapa en la que no son pocos los que ven reducidos sus números de estudiantes en general y de becarios en particular.

\section{¿Algo nuevo en el horizonte?}

En ese escenario es que se llega al cambio de gobierno en diciembre de 2019, luego de la derrota del intento de reelección del presidente Macri en la primera vuelta de las elecciones de octubre de $2019^{27}$. La llegada de Alberto Fernández a la presidencia se presenta como el fin del ciclo de gobierno descrito y abre un escenario de potencial reconstitución de algunas de las políticas que se habían dado hasta fines de 2015.

Sin embargo iniciando el segundo semestre de la nueva gestión, y con la abrupta irrupción de la pandemia mundial de COVID-19 que ha puesto entre paréntesis a buena parte de la administración pública, todo parece demasiado prematuro para poder analizar los rumbos que tomarán las políticas hacia el sector universitario en los próximos años.

Como ya ha pasado en otras múltiples ocasiones en la historia argentina reciente, las diversas urgencias que encontró el nuevo gobierno se han mostrado de tal envergadura que no han permitido desarrollar, de momento, una clara agenda de temas para el sistema universitario.

La situación económica muestra una severa recesión, con altos niveles de desempleo, pobreza e inflación, con un abultado endeudamiento público contraído a altas tasas de interés y con vencimientos muy concentrados en el corto plazo. Ello resulta una enorme amenaza con el potencial de condicionar toda la política pública del nuevo gobierno, en especial por las importantes restricciones presupuestarias que promete imponer, incluso en los horizontes más benévolos, como el de un exitoso proceso de renegociación de esos pasivos cuyos capítulos se siguen sucediendo al momento de escribir este artículo ${ }^{28}$.

A ello se le ha sumado la mencionada pandemia de COVID-19 que azota al planeta desde fines del primer trimestre del año 2020, y que ha profundizado la crisis económica argentina, con un enorme aumento del déficit fiscal, producto del incremento del gasto público de emergencia, en especial para sostener salarios y empleos ante los cierres preventivos de buena parte de la economía, combinado con el desplome de la recaudación tributaria por el menor nivel de actividad.

Esto también ha significado la paralización de buena parte de la administración pública, y de las universidades.

Como pocas veces en la historia moderna, las universidades de todo el mundo han interrumpido sus clases presenciales, ce- 
rrando sus instalaciones y comenzado un proceso de reorganización de sus tareas de docencia e investigación, que no podemos prever su fin ni sus consecuencias. En este escenario las universidades argentinas han acompañado estas disposiciones, interrumpiendo la mayor parte de sus actividades cotidianas, y trasladando a modalidades virtuales una parte significativa de sus tareas docentes.

Así la pregunta por la nueva realidad de las universidades argentinas ante el cambio de gobierno remite hoy a un gran signo de interrogación en este escenario de incertidumbre.

Queda claro que la disponibilidad de recursos significativos para destinar a las universidades no parece un escenario muy esperable en el corto y mediano plazo, con lo que no se pueden esperar grandes inversiones para el desarrollo del sector.

Con relación al probable sentido de las políticas de educación superior y de ciencia y tecnología, solo podemos hacer algunas deducciones a partir de ciertas señales, esbozadas imperfectamente hasta el momento.

Lo primero que es perceptible como señal es que se va produciendo un cambio en la retórica hacia la universidad (en especial a las públicas) y a la ciencia.

El nuevo presidente Alberto Fernández se ha ocupado de presentarse constantemente como un profesor universitario de una universidad pública, incluso sosteniendo esa actividad ya asumido en funciones ${ }^{29}$.

Esto busca marcar un contraste importante con el gobierno anterior, en el que la mayor parte del gabinete y el propio presidente eran graduados de universidades privadas y donde parecía primar cierta distancia y desconfianza hacia el sistema público. El segundo eje ha sido la nueva retórica a favor de la ciencia.

La anteriormente degradada Secretaría de Ciencia y Tecnología recuperó el rango de ministerio con la asunción del gobierno el 10 de diciembre de 2019.

Poco después, ya en enero, se anuncia una recomposición por etapas de los montos de los estipendios de las becas doctorales y posdoctorales que otorga el Estado Nacional a través de diversos organismos, reparando una situación de fuertes atrasos acumulados en los años previos.

En febrero de 2020 se reorganiza, con cambio de nombre incluido, la ahora llamada Agencia Nacional de Promoción de la Investigación, el Desarrollo Tecnológico y la Innovación en lo que podría presentarse como una apuesta a darle un mayor apoyo financiero a la investigación.

También en febrero se anuncia el incremento sustancial del número de científicos que ingresan a la Carrera de Investigador Científico del CONICET pasando de los 450 del año precedente a 800 agentes para el 2020 (correspondiente a la convocatoria 2019).

Sin embargo las restricciones presupuestarias señaladas y el inicio de la crisis pusieron en suspenso otras iniciativas de mayor envergadura, desde la actualización de los sueldos del personal de Ciencia y Tecnología, hasta el reinicio de numerosas inversiones en infraestructura pendientes.

Tal vez la última señal previa al brutal cambio de escenario que produjo la irrupción de la pandemia la podamos encontrar en el discurso presidencial de apertura de las sesiones ordinarias del Poder Legislativo, realizado 
el 1 de marzo de 2020, a menos de tres meses de la asunción y casi al filo del inesperado cambio de contexto que se iba a dar pocos días después ${ }^{30}$.

En ese discurso, tradicionalmente inscrito en los de tipo programático, el nuevo presidente destacó que encabezaba "un gobierno de científicos no de CEOs" para marcar una fuerte diferencia con su antecesor. Muy pocas semanas después, conformó un comité de expertos médicos y científicos de consulta permanente, para asesorar al poder ejecutivo en la gestión de la pandemia y la cuarentena dispuesta.

Con relación a las universidades en el mencionado discurso el presidente anunció ante la Asamblea Legislativa, de forma bastante imprevista, su intención de "iniciar el debate" sobre una nueva Ley de Educación Superior (LES).

El discurso no deja muy claro los sentidos de ese debate, más allá de la reafirmación del papel de las universidades en la democratización del conocimiento, y aclarando que la gratuidad y el ingreso irrestricto constituyen sus puntos de partida, pero también insinuando la voluntad de fortalecer la vinculación de las universidades con la comunidad y el sector productivo.

Este anuncio, tal vez el más significativo para el sistema universitario, la principal señal del nuevo gobierno de tomar cartas en el tema y de avanzar en cambios, no ha tenido réplicas posteriores ni del presidente, ni en el ministerio correspondiente.

La situación de excepcionalidad de la pandemia parece haber puesto en suspenso el inicio de estas muy postergadas discusiones ${ }^{31}$.

Recordemos que la LES ya presentada es la legislación vigente desde 1995 para todo el sistema de educación superior argentino.

Se trata de una norma que surge en pleno auge de las políticas neoliberales, y que abrió la puerta, con significativo éxito, para una serie de reformas de las universidades que se han consolidado en este último cuarto de siglo.

Si bien la norma ha sido objeto de innumerables críticas, en especial luego de la crisis de 2001-2, lo que llevó a que en los primeros años del presente siglo todos los bloques partidarios en el Congreso de la Nación hayan tenido sus propios proyectos de reforma de la ley, la realidad fue que nunca se alcanzaron los consensos para avanzar en esos cambios.

Pareciera haber unanimidad para criticar la normativa, pero pocos acuerdos sobre cómo reempla- 
zarla como se ve a lo largo de los tres mandatos presidenciales que se suceden entre 2003 y 2015, donde a pesar de las críticas, no se logra avanzar con una nueva ley para el sector.

La referencia de Fernández, relativamente sorpresiva pero además no retomada posteriormente, abre un abanico de posibilidades que sin dudas, si logra salir del terreno de la mera retórica, será el aporte central de la nueva gestión al sistema universitario.

De momento solo podemos decir que algunas cuestiones como las referidas a las formas de gobierno, al funcionamiento presupuestario, a los mecanismos de ingreso a la docencia (en especial los concursos) y de carrera académica, el rol de la investigación y la extensión, a la internacionalización y la federalización, o los debates sobre calidad y acreditación prometen enormes discusiones y supondrán difíciles consensos si es que se llega a ellos.

\section{Conclusiones}

La universidad argentina cambia y demanda cambios. Son dos cosas diferentes. Lo primero es producto de su propia complejidad que, impactada por la realidad, produce constantes transformaciones. La virtualidad a la que la epidemia ha movido en estos meses es un ejemplo de lo primero. Pero una demanda de cambios debe ser un proceso más profundo, más reflexionado, no una mera adaptación puntual.

Comprometer un debate sobre una nueva ley para el sector parece ir en sintonía con ello. Supone construir consensos difíciles, que deben surgir de las lecturas y los debates críticos sobre lo que se hace y lo que no se hace, sobre lo que resulta deseable y lo que merece una resistencia.

Los tiempos mundiales no parecen ayudar mucho. Ante el proceso de transformación global de la universidad, que la lleva por el sendero de su mercantilización, la universidad argentina ha quedado relativamente al margen, más por inmovilismo que por una decisión reflexiva y consensuada. Abrir la puerta a cambios profundos, más en escenarios de restricción presupuestaria severa, puede ser ir en esa dirección.

Por otro lado, un aprendizaje de las políticas públicas del sector es que, dada la alta autonomía del sistema, los cambios no tienen muchas probabilidades de imponerse "desde arriba". Deben construir consensos y eso suele ir de la mano de incentivos para que se produzcan.

La estrechez presupuestaria que se avizora en los próximos años no parece el mejor escenario para 
encarar esa negociación. Si el gobierno no cuenta con recursos económicos para premiar a los que vayan realizando los cambios que se definan, es probable que los mismos no se adopten, o que solo lo hagan los que despierten acuerdos en las universidades, que por la heterogeneidad planteada, serán los más conservadores.

\section{Notas}

1. En el artículo 75 inc. 19 de la Constitución de la Nación Argentina. Notamos que la inclusión de este tema en los textos constitucionales comienza a devenir de manera frecuente en las últimas décadas, como se puede ver en el art. 3 inc. VII de la Constitución Política de los Estados Unidos Mexicanos con la reforma de 1980, en el art. 207 de la Constitución de la República Federativa de Brasil de 1988, en el art. 69 de la Constitución Política de Colombia de 1991, o en el art. 18 de la Constitución Política del Perú de 1993 por dar algunos ejemplos. Sin embargo el ejercicio de esa autonomía parecería más potente en Argentina por lo modos en que se ha hecho valer en diversas instancias judiciales.

2. En este primer semestre de 2020 podemos ver la profundización de una serie de tendencias económicas preocupantes. En primer lugar, los efectos locales y mundiales de la epidemia de COVID-19 que ha llevado al cierre de buena parte de la economía, y en paralelo una nueva crisis de endeudamiento externo.

3. Los datos oficiales para 2016-2017, últimos disponibles, presentan una tasa bruta universitaria de $38,6 \%$ en un sistema con 131 instituciones universitarias, y donde el subsistema público tiene presencia en todas las 24 jurisdicciones provinciales que integran el país (SPU, 2018). Para ver tasas brutas correspondientes a los cortes censales de 2001 y 2010 , véase Fachelli y López-Roldán (2017).

4. Que ha tenido una extensa trayectoria desde 1949, con algunas interrupciones, pero que se consolidó en las últimas décadas. El tema está considerado en la Ley de Educación Superior (24521) de 1995 y es reafirmado en la Ley 27204 de 2015 (Ley de Implementación efectiva de la responsabilidad del Estado en el nivel de educación superior).

5. Con excepción de los estudios de medicina que han desplegado en diversas instituciones sus propias estrategias de regulación de los ingresantes.

6. Barsky y Corengia (2018). A fines de los 90 el subsector privado captaba $15 \%$ de los estudiantes universitarios, lo que hace que en menos dos décadas su participación se haya elevado casi $50 \%$.

7. Las carreras de abogacía, medicina, contador público, administración de empresas y psicología.

8. Son ejemplos de ello el Programa Nacional de Becas Bicentenario, lanzado en 2009 para fomentar el estudio de carreras científicas y tecnológicas, o el Plan Estratégico de Formación de Ingenieros 2012-2016 que vino a complementar parcialmente al primero.

9. Por ejemplo Chiroleu (2009), García de Fanelli (2015).

10. Marquina (2016), Claverie (2015).

11. Los últimos datos de la SPU correspondientes a 2016-2017 señalan que $11,3 \%$ de los docentes universitarios tienen dedicaciones exclusivas, y $18,4 \%$ semiexclusivas que se corresponden con 40 y 20 horas semanales respectivamente. 
12. El cuatrienio presidencial se extiende hasta diciembre de 2019.

13. La coalición también está integrada por la Unión Cívica Radical, partido con fuerte ascendencia sobre varias gestiones de universidades nacionales.

14. Una devaluación de la moneda nacional de más de $30 \%$ en un día, más la sostenida política de reducción de una serie de subsidios públicos a las tarifas de agua, gas, electricidad, que significaron un importante aumento de las mismas (del orden de $250 \%$ en algunas de ellas).

15. Los cálculos presentados por el gremio CONADU (Federación Nacional De Docentes Universitarios) señalan una caída del poder adquisitivo de los salarios docentes entre diciembre del 2015 y diciembre de 2016, primer año del nuevo gobierno, de 8,28\%.

16. Aunque es necesario aclarar que los acatamientos a muchas de esas medidas de fuerza convocadas por los gremios universitarios no fueron homogéneos.

17. Un apartado del Decreto 1023/2001.

18. Solo subsisten una minoría debidamente justificada por los ministros correspondientes.

19. En ese momento había 53 universidades públicas en funcionamiento y dos creadas, pero aún sin estudiantes. De las que estaban desarrollando actividades la única que queda al margen de la denuncia es la Universidad de la Defensa Nacional, fundada en 2014, y dependiente del Ministerio de Defensa y no del de Educación.

20. Hemos trabajado este tema en Unzué y Rovelli (2017) y Unzué y Emiliozzi (2017).

21. El científico Lino Barañao, que había sido el primer ministro del área durante el anterior gobierno de Cristina Fernández (a partir de 2007 el área de ciencia y tecnología es jerarquizada pasando a rango de ministerio) tiene el privilegio inédito de continuar en ese cargo en el gobierno de Macri, como claro signo de una voluntad de continuidad. A pesar de ello, y en medio de una reestructuración general de septiembre de 2018, el propio Macri vuelve a rango de Secretaría al Ministerio, ya con una política más abierta de reducción de su relevancia.

22. Los nuevos nombramientos representan $2 \%$ de los cargos de dedicación exclusiva existentes, y $0,25 \%$ del total de cargos docentes de las universidades nacionales.

23. Resolución Ministerial 1870 del 28 de octubre de 2016.

24. Esto sería 0,01\% del total de estudiantes universitarios.

25. Resolución Ministerial núm. 1016 de fecha 13 de abril de 2018.

26. La Comisión Asesora establecida en el artículo 9 de la resolución se integra con cuatro representantes por el Consejo Interuniversitario Nacional (CIN), dos por el Consejo de Rectores de Universidades Privadas (CRUP), dos por la SPU y dos por la Secretaría de Ciencia y Tecnología (SECYT).

27. Macri es el primer presidente, desde el retorno a la democracia, que intenta su reelección sin éxito.

28. A comienzos de junio de 2020 no se puede descartar ninguna hipótesis sobre este punto, pero sí queda claro que en cualquier escenario el endeudamiento con actores privados y con organismos multilaterales de crédito (en especial el Fondo Monetario Internacional) condicionará las posibilidades de inversión futuras en el sector universitario.

29. En una cierta sobreactuación, ha concurrido varias veces a dictar clases a la Facultad de Derecho de la Universidad de Buenos Aires en su rol de presidente, lo que fue ampliamente cubierto por la prensa. Sobre este tema de la universidad como legitimante 
de la acción política hemos trabajado en Unzué (2016).

30. El decreto que establece el aislamiento social preventivo y obligatorio se dicta el 19 de marzo para entrar en vigencia el día 20.

31. Aunque el tema ha sido inevitablemente objeto de discusión tanto entre miembros del Consejo Interuniversitario Nacional (CIN) como del CRUP.

\section{Referencias}

Barsky, O. y A. Corengia comps. (2018). La Ley de Educación Superior: impactos, desafíos e incertidumbres. Buenos Aires: Teseo.

Claverie, J. (2015). "Trabajo y condiciones de carrera para los docentes de las universidades nacionales de la Argentina. El problema de la movilidad" en Trabajo y Sociedad. Núm. 25. Invierno 2015, Universidad Nacional de Santiago del Estero, Santiago del Estero.

Constitución de la Nación Argentina disponible en http://servicios.infoleg.gob.ar/infolegInternet/ anexos/0-4999/804/norma.htm

Chiroleu, A. (2009). "La democratización del acceso a la universidad: de la ampliación de oportunidades a la inclusión”. En A. Chiroleu y M. Marquina. (comp.). A 90 años de la Reforma Universitaria: memorias del pasado y sentidos del presente. riepesal. San Miguel: Coedición ungs-Prometeo Libros.

Decreto 1023/2001 disponible en https://www.argentina.gob.ar/normativa/nacional/decreto-1023-2001-68396

Fachelli, S. y P. López-Roldán (2017). Análisis del sistema universitario argentino. Una propuesta inicial de indicadores. Barcelona, Universitat Autònoma de Barcelon. Disponible en http:// ddd.uab.cat/record/171528

García de Fanelli, A. M. (2015). "La cuestión de la graduación en las universidades nacionales de la Argentina: Indicadores y políticas públicas a comienzos del siglo XXI”. Facultad Latinoamericana de Ciencias Sociales, Propuesta Educativa 1 43; 6-2015; 17-31.

Ley de Educación Superior 24521/95. Disponible en http://servicios.infoleg.gob.ar/infolegInternet/ anexos/25000-29999/25394/texact.htm

Ley 27204/15 De implementación efectiva de la responsabilidad del estado en el nivel de educación superior. Disponible en https://www.argentina.gob.ar/normativa/nacional/ley-27204$254825 /$ texto

Marquina, M. (2016). Yo te evalúo, tú me evalúas... Estado, profesión académica y mercado en la acreditación de carreras en la Argentina. Buenos Aires, Eudeba.

Resolución Ministerial 1870 del 28 de octubre de 2016. Disponible en http://servicios.infoleg.gob. ar/infolegInternet/anexos/265000-269999/267158/norma.htm

Resolución Ministerial 1016 del 13 de abril de 2018. Disponible en https://www.boletinoficial.gob. ar/detalleAviso/primera/181881/20180418

SPU (2018). Síntesis de información de estadísticas universitarias. Buenos Aires, Secretaría de Políticas Universitarias, Ministerio de Educación.

Unzué, M. y L. Rovelli (2017). “Cambios, tendencias y desafíos de las políticas científicas recientes en las universidades nacionales de la Argentina”, Revista Tla-Melaua. Año 11, Núm. 42, abril-septiembre 2017, Facultad de Derecho y Ciencias Sociales de la Benemérita Universidad Autónoma de Puebla, México. Disponible en http://www.scielo.org.mx/scielo.php?script=sci_arttext\&pid=S1870-69162017000200242\&lng=es\&nrm=iso

Unzué, M. y S. Emiliozzi (2017). "Las políticas públicas de Ciencia y Tecnología en Argentina: un balance del periodo 2003-2015”, Revista Temas y Debates, Facultad de Ciencia Política y Relaciones Internacionales de la Universidad Nacional de Rosario. Año 21, Núm. 33, enero-junio 2017, disponible en: http://www.temasydebates.unr.edu.ar/index.php/tyd/article/view/353

Unzué, M. (2016). Los universitarios y la política. Buenos Aires, Imago Mundi. 